\title{
A reply from Paradies
}

SIR - My 1970 paper is not a misrepresentation because:

(1) The crystal diffracts well and does not deteriorate as long as the temperature is below $4^{\circ} \mathrm{C}$. But within $4 \mathrm{~h}$ of increasing the temperature from $4^{\circ} \mathrm{C}$ to $21^{\circ} \mathrm{C}$ the vapour pressure of $t$-butanol/dioxane increases more than 10-fold so that the tRNA crystal dissolves. Therefore, the loss of the high angle reflections in Fig. 3 after $3 \mathrm{~h}$ of X-ray exposure at $21^{\circ} \mathrm{C}$ (the crystal was actually at $21^{\circ} \mathrm{C}$ for more than $4 \mathrm{~h}$ ) is not unexpected. An additional cause of crystal destruction is damage by $\mathrm{X}$-ray-induced tertiary butanol radicals and short-lived dioxane radicals. The statements by Hendrickson et al. of the "short X-ray exposure" and the beam stop shadow and the expression "suddenly be completely destroyed", are unfounded. Even for obtaining the X-ray picture, shown in Fig. 2, 90 minutes were necessary, as stated.

(2) The tRNA crystal was definitely not a small one; for obtaining the general cell dimensions, a crystal size of $0.1 \mathrm{~mm}$ would be sufficient for a macromolecule with a molecular weight of 25,000 . Since the intensity is roughly proportional to the diffraction volume of the crystal, the reported crystal size of $0.2 \times 0.15 \times 0.2 \mathrm{~mm}$ yields a diffracting volume six times that needed for getting the cell dimensions and, if it is stable at $21^{\circ} \mathrm{C}$, has almost the quality, from the point of intensity distribution of the spots and diffracting volume, for high resolution $\mathrm{X}$-ray analysis.

(3) Hendrickson et al. recalculate from Fig. 2 of ref. 1 the ratio of the spacings in the nets as 1.09 but that is meaningless because the magnification, not indicated in Fig. 2, is different in the horizontal and vertical planes. In the horizontal plane it is $\times 1.45-1.48$ and in the vertical plane it is $\times 1.35-1.37$, revealing a ratio of about 1.085-1.09. Multiplication of the ratio of the net spacings of the X-ray pattern of Fig. 2 with the ratios of the enlargement, $1.09 \times 1.085$, yields 1.18 (ref.3), which is consistent with the reported parameters. Since the film-to-specimen distance is 75 $\mathrm{mm}$, and not $60 \mathrm{~mm}$, as Hendrickson et al. impute, the dimensions of the net are calculated to $94 \AA$ and $80 \AA$ in the low angle region in the $k h 1$ plane to $94.05 \AA$ and $80.21 \AA$, and in the $h k 2$ plane to approximately $94 \AA$ and 79-80 $\AA$. However, a calculation of the net, assuming $F=60$ $\mathrm{mm}$, leads to values of $75 \pm 1.5 \AA$ and 64.2 $\pm 2.0 \AA$, respectively. Neither value for $F$ ( $75 \mathrm{~mm}$ or $60 \mathrm{~mm}$ ) is consistent with those for a crystal of human carbonic anhydrase B with $a=74.4 \AA, b=81.4 \AA$ and $c=37.8$ $\AA$. Spacings in the first annulus were measured to be 1.228 horizontally and 1.440 vertically. The third axis is calculated to be 34-35 $\AA$, with $F=75 \mathrm{~mm}$, which also is not consistent with the $c$-dimension of carbonic anhydrase.

(4) The X-ray pattern does not show complete $\mathrm{mm}$ symmetry; this is more pronounced at higher levels, which might be due to the misalignment and slipping of the crystal, causing the perturbations that are clearly to be seen. Furthermore, the unit cell angles in the upper levels are not all $90^{\circ} \mathrm{C}$. Therefore, this pattern and the intensity distribution in the $h k 1$ and $h k 2$ layers are not those of a crystal of human carbonic anhydrase $\mathbf{B}$. Nor would it have yielded the observed $h k 0$ intensity distribution, including the unusual distribution of dots coming from the $\beta$ radiation. Although the unit cell is apparently orthorhombic and there is some indication of $\mathrm{mm}$ symmetry the possibility exists that the crystal symmetry with respect to higher resolution (see above) is monoclinic.

In conclusion: cell dimensions, crystal stability and solubility, including temperature dependence, are consistent with a crystal of tRNA.

With respect to my other papers:

(1) The net of the diffraction patterns for the seryl-tRNA crystals (2) was calculated by a colleague in Uppsala, Sweden, and it revealed almost the same result as that previously obtained from a powder pattern, which was also included in that papers.

(2) There are no inconsistencies between the measured $d$-spacings and the figure in my 1968 paper ${ }^{3}$.

(3) The crystals of valyl-tRNA synthetase ${ }^{4}$ are not a misrepresentation of a crystal of human carbonic anhydrase $C$. The crystal in Fig. 4 is vertically misaligned from the (001) axis, caused by the crystal having slipped in the capillary during exposure. The mentioned orthorhombic crystals of the synthetase are not documented, only the cell dimensions. And, again, magnifications were not stated on the figure.

(4) My contribution to the characterization of crystals of D-ribulose 1,5-diphosphate carboxylase ${ }^{5}$ was only the determination of the crystal density. The electron micrographs were obtained by my co-authors and the optical diffraction pattern was obtained with the facilities of Dr Räuber, Fritz-Haber-Institut, BerlinWest. Assuming a molecular weight of $515,000-550,000$, all reported results in this paper are correct, including the magnitude of $V_{M}$, with no assumption made of the number stoichiometry of the large and small subunits.

(5) The very unfortunate mistake by which the electron micrograph and its optical diffraction patterns of ref.5 were used again in my paper on spinach chloroplast coupling factor ${ }^{6}$ was corrected as soon as the error was brought to my attention ${ }^{7}$. The results in both my 1979 paper $^{6}$ and my 1980 paper $^{8}$ were compared with other physical measurements (see also ref.6).

\section{$D-1000$ Berlin 45, \\ FRG}

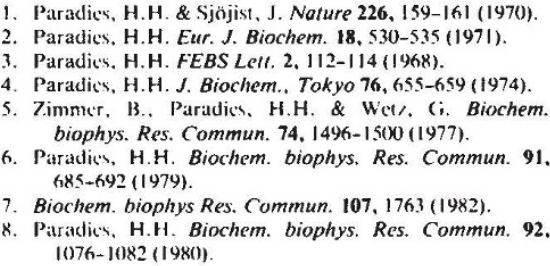

1. P'aradies, H.H. \& Sjöjist, J. Noture 226, 159-|61 (1970). 2. Paradies, H.H. Eur. J. Biochem. 18, 530-535 (1971).

. Paradics, H.H. FEBS Lell, 2, 112-114 (1968).

Paradics, H.H.J. Biochem. Tokyo 76, 655-659 (1974).

Zimmer, B., Paradics, H.H. \& Wet, (;. Biochem. Zimmer, B., Paradics, H.H. \& Wet/, (i. B
biophys. Res. Commun. 74, 1496-1500 (1977).

. Paradies, H.H. Biochem. biophys. Res. Commun. 91. $685-692(1979)$.

Biochem. biophys Res. Commun. 107, 1763 (1982)

. Paradics, H.H. Biochem. biophys. Res. Commun. 92. $1076-1082(1980)$.

\section{Evolution by numbers}

SIR - In the discussion in your columns about the application of quantitative methodology based on the study of evolutionary processes to the analysis of the development of human culture ${ }^{1,2}$, there is an unquestioned assumption on both sides of that issue that quantitative theory, as expounded by practitioners such as Fisher, Haldane, Wright, Cavalli-Sforza and Maynard Smith, has been successful in illuminating and explaining the process of biological evolution and the genetic relationships between species. As far as I know, there is no evidence to support this assumption. Indeed, there is a vast number of observations unaccounted for in the extant quantitative evolutionary theories. Many of these observations (inducible mutation systems ${ }^{3}$, rapid genomic changes involving mobile genetic elements ${ }^{4}$, programmed changes in chromosome structure ${ }^{5-8}$ ) challenge the most fundamental assumptions which these evolutionary theories make about the mechanisms of hereditary variation and the fixation of genetic differences.

As a practising geneticist, I am frequently astonished by the ease with which population theorists assume complex (and therefore troublesome) phenomena out of existence, no matter how solid the documentation. Perhaps they should set their own house in order and come to terms with what genetics and molecular biology have to teach them about possible mechanisms of biological evolution before they try to save anthropology from the anthropologists.

JAMES A. SHAPIRO

\section{Department of Microbiology} University of Chicago, USA

I. each. F. Nature 291, 267 (1981).

. Marks, I. et al. Nature 302, 15 (1983).

Within, F. Bact. Rer. 40, 869 (1976)

Shapiro, J. (edi.) Mobile Genetic Elements (Acadenic, New York, 1983).

Toncgawa et al. Cold Spring Harb. Symp. quant. Biol. 45. $839(1981)$

6. Nanncy. D.1. Experimental Ciliatology (I. Wiky, New York. 1980).

7. Becrman. S. Chromosoma (Berl.) 60, 297 (1977).

8. Boneri, T. S. B. ges. Morph. Phusiol. (Munchen) 8. $14(1 \times 92)$. 